\title{
Subcutaneous Uptake on [18F]Florbetaben PET/CT: a Case Report of Possible Amyloid-Beta Immune-Reactivity After COVID-19 Vaccination
}

\author{
Riccardo Laudicella ${ }^{1,2,3}$ (1) Irene Andrea Burger ${ }^{3,4}\left(\right.$ C) $\cdot$ Francesco Panasiti $^{1,2} \cdot$ Costanza Longo $^{1,2} \cdot$ Salvatore Scalisi ${ }^{1}$. \\ Fabio Minutoli $^{2} \cdot$ Sergio Baldari ${ }^{2}$ (D) Luigi Maria Edoardo Grimaldi ${ }^{5} \cdot$ Pierpaolo Alongi $^{1}$ (I)
}

Accepted: 1 September 2021 / Published online: 14 September 2021

(c) The Author(s) 2021

\begin{abstract}
Introduction Large-scale worldwide COVID-19 vaccination programs are being rapidly deployed, and high-risk patients with comorbidity are now receiving the first doses of the vaccine. Physicians should be, therefore, aware of new pitfalls associated with the current pandemic vaccination program, also in the case of $\left[{ }^{18} \mathrm{~F}\right]$ Florbetaben PET/CT.

Case Presentation

We described the first image of $\left[{ }^{18} \mathrm{~F}\right]$ Florbetaben PET/CT in the evaluation of a 70-year-old male with suspicious Alzheimer disease and unclear history of heart disease. We detailed the diagnostic imaging PET/CT workup with different findings.

Conclusion In this case, $\left[{ }^{18} \mathrm{~F}\right]$ Florbetaben PET/CT can demonstrate potential beta-amyloid immune-reactivity and deposition associated with the current COVID-19 pandemic vaccination programs.
\end{abstract}

Keywords COVID-19 $\cdot$ Amyloid $\cdot$ PET/CT $\cdot$ Alzheimer $\cdot$ Florbetaben $\cdot$ Vaccination

\section{Introduction}

Large-scale worldwide COVID-19 vaccination programs are being rapidly deployed, and high-risk patients with comorbidity are now receiving the first doses of the vaccine. Physicians should be, therefore, aware of new pitfalls associated

This article is part of the Topical Collection on COVID-19

Riccardo Laudicella

riclaudi@hotmail.it

1 Nuclear Medicine Unit, Fondazione Istituto G. Giglio, Contrada Pietrapollastra Pisciotto, Cefalù (Palermo), Italy

2 Department of Biomedical and Dental Sciences and of Morpho-Functional Imaging, Nuclear Medicine Unit, University of Messina, Messina, Italy

3 Department of Nuclear Medicine, University Hospital Zurich, University of Zurich, Zurich, Switzerland

4 Department of Nuclear Medicine, Cantonal Hospital of Baden, Baden, Switzerland

5 Neurology Unit, Fondazione Istituto G. Giglio, Contrada Pietrapollastra Pisciotto, Cefalù (Palermo), Italy with the current pandemic vaccination program, also in the case of $\left[{ }^{18} \mathrm{~F}\right]$ Florbetaben PET/CT.

\section{Case Presentation}

We report the case of a 70-year-old male who underwent $\left[{ }^{18} \mathrm{~F}\right]$ Florbetaben PET/CT for suspected Alzheimer disease (AD) 1 day after the administration of the first dose of Pfizer-BioNTech COVID-19 vaccine in the right arm in absence of any related symptoms. The patient was also referred with a concomitant unclear history of heart disease (hypertensive disease and initial signs of heart failure with suspicious of cardiac amyloidosis), and therefore, the thorax was also included in the acquisition's field due to [18F] Florbetaben PET potential utility in the diagnostic workup of cardiac amyloidosis [1,2]. A moderate amyloid burden on the bilateral frontal and parietal brain cortex in the absence of cardiac beta-amyloid deposition was identified, referable to as the presence/development of AD. However, subcutaneous uptake on the vaccination site in the right arm's deltoid region and focal uptake next to an ipsilateral axillary lymph node were noted. Tracer injection was via the left 


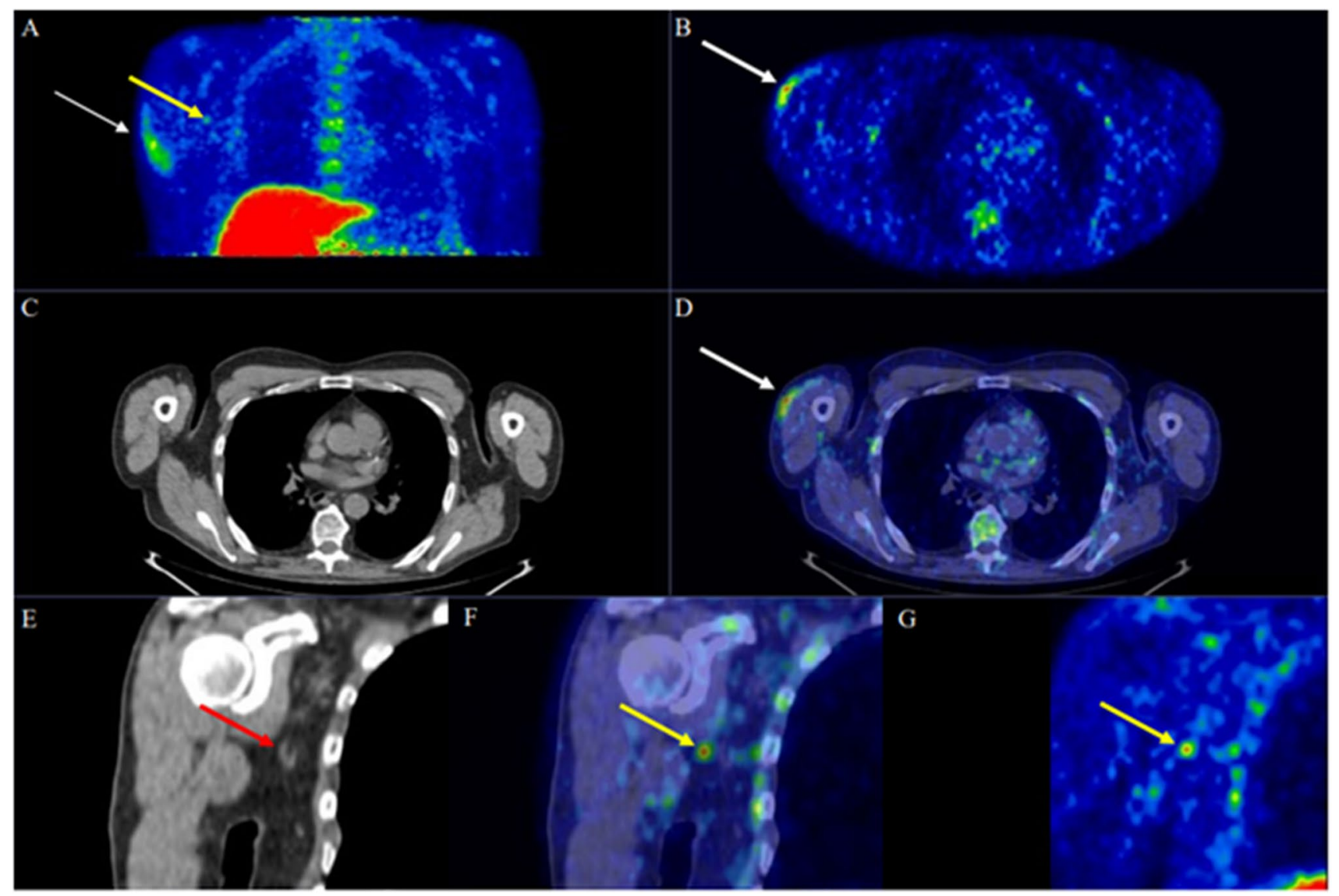

Fig. $1\left[{ }^{18}\right.$ F]Florbetaben PET/CT: MIP (A), PET (axial-B, coronal$\mathbf{G})$, CT (axial-C, coronal-E), PET/CT (axial-D, coronal-F) images demonstrated ill-defined uptake in the right arm's subcutaneous tissues (SUVmax 5.6; white-arrows) and next to a possible right-axillar

antecubital fossa, hence not a potential cause. In Fig. $1,\left[{ }^{18} \mathrm{~F}\right]$ Florbetaben MIP (A), PET (axial-B, coronal-G), CT (axialC, coronal-E), PET/CT (axial-D, coronal-F) images demonstrated ill-defined uptake in the right arm's subcutaneous tissues (SUVmax 5.6; white-arrows) and next to a possible right-axillar lymph node (SUVmax 4.75; yellow-arrows) evident on low-dose CT scan without breathing control (red arrows). We assume that the subcutaneous and the potential lymph node uptake might be related to an induced inflammation with peptides deposition, as amyloid-beta peptides are involved in the systemic inflammatory process such as in the physiopathology of $\mathrm{AD}$, and chronic, low-level systemic inflammation may exacerbate the $A \beta$ deposition [3-6]. Such induced inflammation, already demonstrated with several tracers [7-9], may be further enhanced in AD patients by the amyloid-beta presence. In this sense, Hsu et al. observed in a preclinical study that the intracellular immunoreactivity was significantly increased by the SARS-CoV-2 pseudovirus in the presence of $A \beta_{1-42}$ (a strong indicator of $A D$ with high affinity for SARS-CoV-2 spike protein $\mathrm{S} 1$ subunit) compared lymph node (SUVmax 4.75; yellow-arrows) evident on low-dose CT scan without breathing control (red arrows). Reprinted with permission from Nuclear Medicine Unit, Fondazione Istituto G. Giglio, Cefalù (Palermo), Italy.

to SARS-CoV-2 pseudovirus alone. Interestingly, they also observed that $\mathrm{A} \beta_{1-42}$ significantly increased SARS-CoV-2 infectivity and that the clearance of $A \beta_{1-42}$ can be reduced during SARS-CoV-2 infection [10]. Similar findings with other radiopharmaceuticals were recently described [7-9], but this is the first case to show that also $\left[{ }^{18} \mathrm{~F}\right]$ Florbetaben PET/CT can demonstrate immune-induced findings, also amplified by the beta-amyloid presence, associated with the current COVID-19 pandemic vaccination programs, being a potential finding on whole-body protocols for the assessment of cardiac/systemic amyloidosis.

\section{Conclusion}

$\left[{ }^{18} \mathrm{~F}\right]$ Florbetaben PET/CT can demonstrate immune-induced findings, also amplified by the beta-amyloid presence, associated with the current COVID-19 pandemic vaccination programs. 
Author Contribution RL, PA: conceptualization and original draft writing; GLME: conceptualization; IAB, FM, SB: review and editing; SS, FP, CL: data curation.

Funding Open access funding provided by University of Zurich.

Data Availability Data are available for bona fide researchers who request it from the authors.

\section{Declarations}

Ethics Approval and Informed Consent All procedures performed involving the human participant were under the ethical standards of the institutional and/or national research committee and with the 1964 Helsinki declaration and its later amendments or comparable ethical standards. Informed consent was obtained from the patient.

Consent to Participate Informed consent was obtained from the patient.

Consent for Publication The consent for publication was obtained from the patient.

Competing Interests The authors declare no competing interests.

Open Access This article is licensed under a Creative Commons Attribution 4.0 International License, which permits use, sharing, adaptation, distribution and reproduction in any medium or format, as long as you give appropriate credit to the original author(s) and the source, provide a link to the Creative Commons licence, and indicate if changes were made. The images or other third party material in this article are included in the article's Creative Commons licence, unless indicated otherwise in a credit line to the material. If material is not included in the article's Creative Commons licence and your intended use is not permitted by statutory regulation or exceeds the permitted use, you will need to obtain permission directly from the copyright holder. To view a copy of this licence, visit http://creativecommons.org/licenses/by/4.0/.

\section{References}

1. Law WP, Wang WY, Moore PT, Mollee PN, Ng AC. Cardiac amyloid imaging with 18F-florbetaben PET: a pilot study. J Nucl Med. 2016;57:1733-9. https://doi.org/10.2967/jnumed.115.169870.

2. Baratto L, Park SY, Hatami N, Gulaka P, Vasanawala S, Yohannan TK, Herfkens R, Witteles R, Iagaru A. 18F-florbetaben whole-body PET/MRI for evaluation of systemic amyloid deposition. EJNMMI Res. 2018;8:66. https://doi.org/10.1186/ s13550-018-0425-1.

3. Bucerius J, Barthel H, Tiepolt S, Werner P, Sluimer JC, Wildberger JE, Patt M, Hesse S, Gertz HJ, Biessen EA, Mottaghy FM, Sabri O. Feasibility of in vivo $18 \mathrm{~F}$-florbetaben PET/ MR imaging of human carotid amyloid- $\beta$. Eur J Nucl Med Mol Imaging. 2017;44:1119-28. https://doi.org/10.1007/ s00259-017-3651-2.

4. Kokjohn TA, Van Vickle GD, Maarouf CL, Kalback WM, Hunter JM, Daugs ID, Luehrs DC, Lopez J, Brune D, Sue LI, Beach TG, Castano EM, Roher AE. Chemical characterization of pro-inflammatory amyloid-beta peptides in human atherosclerotic lesions and platelets. Biochim Biophys Acta. 2011;181:1508-14. https:// doi.org/10.1016/j.bbadis.2011.07.004.

5. Braak H, Braak E. Neuropathological staging of Alzheimerrelated changes. Acta Neuropathol. 1991;82:239-59. https://doi. org/10.1007/BF00308809.

6. Oberlin LE, Erickson KI, Mackey R, Klunk WE, Aizenstein H, Lopresti BJ, Kuller LH, Lopez OL, Snitz BE. Peripheral inflammatory biomarkers predict the deposition and progression of amyloid- $\beta$ in cognitively unimpaired older adults. Brain Behav Immun. 2021;21:S0889-1591. https://doi.org/10.1016/j.bbi.2021. 03.015 .

7. Eifer M, Eshet Y. Imaging of COVID-19 vaccination at FDG PET/ CT. Radiology. 2021;299(2):E248.

8. Treglia G, Cuzzocrea M, Giovanella L, Elzi L, Muoio B. Prevalence and significance of hypermetabolic lymph nodes detected by 2-[18F]FDG PET/CT after COVID-19 vaccination: a systematic review and a meta-analysis. Pharmaceuticals. 2021;14:762. https://doi.org/10.3390/ph14080762.

9. Nawwar AA, Searle J, Singh R, Lyburn ID. Oxford-AstraZeneca COVID-19 vaccination induced lymphadenopathy on [18F]Choline PET/CT - not only an FDG finding. Eur J Nucl Med Mol Imaging. 2021;4:1-2. https://doi.org/10.1007/ s00259-021-05279-2.

10. Hsu JTA, Tien C-F, Yu G-Y, Shen S, Lee Y-H, Hsu P-C, Wang Y, Chao P-K, Tsay H-J, Shie F-S. The effects of A $\beta 1-42$ binding to the SARS-CoV-2 spike protein S1 subunit and angiotensinconverting enzyme 2. Int J Mol Sci. 2021;22:8226. https://doi. org/10.3390/ijms22158226.

Publisher's Note Springer Nature remains neutral with regard to jurisdictional claims in published maps and institutional affiliations. 